\title{
Ultrafast Elemental and Oxidation-state Mapping of Haematite by 4D Electron Microscopy
}

\author{
Zixue Su, "J. Spencer Baskin, "Wuzong Zhou, ${ }^{*, \dagger}$ John M. Thomas, ${ }^{*, t}$ and Ahmed H. Zewailll, \\ " Physical Biology Center for Ultrafast Science and Technology, Arthur Amos Noyes Laboratory of Chemical Physics, Cali- \\ fornia Institute of Technology, Pasadena, CA 91125, USA \\ ${ }^{\dagger}$ School of Chemistry, University of St Andrews, St Andrews, Fife KY16 9ST, UK \\ * Department of Materials Science and Metallurgy, University of Cambridge, Cambridge CB3 0FS, UK. \\ $\S$ Deceased, 2nd August, 2016.
}

\begin{abstract}
We describe a new methodology that sheds light on the fundamental electronic processes that occur at the sub-surface regions of inorganic solid photocatalysts. Three distinct kinds of microscopic imaging are used that yield spatial, temporal and energyresolved information. We also carefully consider the effect of photon-induced near-field electron microscopy (PINEM), first reported by Zewail et al. in 2009. The value of this methodology is illustrated by studying afresh a popular and viable photocatalyst, haematite, $\alpha-\mathrm{Fe}_{2} \mathrm{O}_{3}$, that exhibits most of the properties required in a practical application. By employing high-energy electron-loss signals (of several hundred $\mathrm{eV}$ ), coupled to femtosecond temporal resolution as well as ultrafast energy-filtered transmission electron microscopy in $4 \mathrm{D}$, we have, inter alia, identified $\mathrm{Fe}^{4+}$ ions that have a lifetime of a few picoseconds, as well as associated photoinduced electronic transitions and charge transfer processes.
\end{abstract}

\section{INTRODUCTION}

Electron energy loss spectroscopy (EELS) in transmission electron microscopy (TEM) is a powerful nanoscale probe providing a wealth of information including atomic composition, chemical bonding, valence state and spin state. ${ }^{1}$ Energyfiltered TEM (EFTEM), as a variant of EELS, combines the chemical composition with the spatial information thus enabling the location of specific elements characterized by specific energy loss in sample at an atomic scale, and with sub eV resolution in elemental mapping. ${ }^{2}$ However, its temporal resolution has hitherto been limited by the speed of the acquisition time of the detector, which usually takes seconds to obtain a reasonably good elemental map in the high energy loss region.

With high resolution in space, time and energy associated with 4D-EM, the dynamics of chemical bonding of nanoscale graphite under photon excitation have been monitored in previous works using femtosecond EELS (FEELS) in the energy range from 0 to $50 \mathrm{eV}$ for the plasmon region, ${ }^{3,4}$ and around 300 $\mathrm{eV}$ for the carbon K-edge core-loss region. ${ }^{5}$ Since the cross-section for inelastic scattering decays with increasing energy loss in a power-law dependence of $\mathrm{AE}^{-\mathrm{r}}$, where $\mathrm{A}$ is a constant, $\mathrm{E}$ the energy loss and $\mathrm{r}$ the inverse power law exponent, ${ }^{1}$ the dramatically weaker signals make it much more challenging to measure the magnitude of the core-loss edge, when the energy loss is high. However, for the first-row transition metals that are of intense interest in photocatalysis, their L-edges corresponding to the $2 \mathrm{p} \rightarrow 3 \mathrm{~d}$ transition are located in the range of $500 \mathrm{eV}$ to $1000 \mathrm{eV}$, and these edges are vital in the determination of spin state, oxidation state and chemical environment. Therefore, extending the FEELS to this region is of fundamental interest for a deeper understanding of the photocatalytic processes in the relevant materials.
A primary example is the splitting of water by a photoelectrochemical method using solar radiation, which is a viable means of producing hydrogen, a clean and storable energy of the future. ${ }^{6-8}$ Over the last two decades haematite $\left(\alpha-\mathrm{Fe}_{2} \mathrm{O}_{3}\right)$ has become one of the most popular and attractive photocatalysts for photoelectrochemically splitting water by solar radiation due to its suitable valence band position and visible light-active bandgap $(\sim 2.1 \mathrm{eV})$, chemical stability, earth-abundance and environmental compatibility. ${ }^{9,10}$ It has been found that the short hole diffusion length is a main disadvantage, hampering the photoelectrochemical performance of haematite. ${ }^{11}$ To date, however, the nature of the photoinduced holes under visible light excitations is still unclear owing to the lack of suitable techniques which can capture these holes before they recombine with electrons in a very short time.

Femtosecond transient absorption spectroscopy (TAS) has been employed to study the photogenerated carrier dynamics, $^{12,13}$ and the related technique ${ }^{14}$ of time-resolved X-ray absorption spectroscopy (XAS) has been employed to study the photoinduced structural and electronic dynamics of a series of transition metals including iron at K-, L- and M-edges. ${ }^{14-20}$ Despite its importance in providing moderate temporal and energy resolution, XAS is not ideal for the in-situ study of a single nanoparticle, due to the low interaction cross-section of X-rays with matter in a very small specimen volume. Besides, none of these techniques can provide in-situ information, pertaining to the local ultramicrostructures of the specimen.

In the present work, we report the in-situ studies of the temporal change of the valence state of iron in nanoscale haematite induced by an ultrashort $519 \mathrm{~nm}$ laser pulse using TEM-FEELS. This temporal change of the valence state of iron on the scale of femtoseconds to picoseconds has been further mapped out by 
iron selected imaging using the inelastic scattering of electrons with an energy loss around $709.5 \mathrm{eV}$ corresponding to the $\mathrm{Fe}$ $\mathrm{L}_{3}$-edge. The unambiguous detection, by both spectroscopy and elemental mapping, of charge transition from $\left(\mathrm{Fe}^{3+}-\mathrm{Fe}^{3+}\right)$ to $\left(\mathrm{Fe}^{2+}-\mathrm{Fe}^{4+}\right)$ demonstrates a prototypical case for the development of 4D elemental mapping, which can be regarded as an important application of 4D electron microscopy.

\section{RESULTS AND DISCUSSION}

FEELS study of the change of iron oxidation-state in haematite. The conventional wisdom for the photoexcitation mechanism is that visible light absorption in haematite causes the indirect $\mathrm{Fe}^{3+} \mathrm{d}-\mathrm{d}$ transition, while the direct ligand to metal (O $2 \mathrm{p} \rightarrow \mathrm{Fe} 3 \mathrm{~d}$ ) charge transfer occurs only when the photon energy is higher than $3.2 \mathrm{eV},{ }^{12,17}$ consistent with the hypothesis of two types of photogenerated holes $\left(\mathrm{Fe}^{4+}\right.$ and $\left.\mathrm{O}^{-}\right)$in the photooxidation of water. ${ }^{21,22}$ However, theoretical studies showed that the lowest unoccupied energy states of haematite are mostly made of Fe $3 \mathrm{~d}$, while the valence band is strongly hybridized and mostly $\mathrm{O} 2 \mathrm{p}$ in character. $^{23,24}$ resulting in a strong $\mathrm{O} 2 \mathrm{p}$ characteristic highest occupied orbital. The ligand to metal transition is also expected to occur in the region $1.55-2.82 \mathrm{eV},{ }^{25,26}$ implying a possible significant $\mathrm{O} 2 \mathrm{p} \rightarrow \mathrm{Fe} 3 \mathrm{~d}$ transition under excitation by visible light. Although the photoholes play a vital role in the photooxidation process, it has not hitherto proved possible to settle the above controversy, owing to the inability to distinguish the two types of photoholes $\mathrm{Fe}^{4+}$ and $\mathrm{O}^{-}$, as well as the related charge transfer processes that are depicted in Figure $1^{27}$

The Fe L-edge core-loss EELS corresponds to the excitation of an $\mathrm{Fe} 2 \mathrm{p}$ core electron in haematite to an unoccupied $3 \mathrm{~d}$ orbital with an energy loss of over $700 \mathrm{eV}$ (Figure 1e and 1f). Its peak position and shape are extremely sensitive to the Fe oxidation state. With FEELS using 4D-EM, it is possible to follow the ultrafast change of iron oxidation state in photoexcited haematite, and to identify the actual electron transfer process. The haematite particles used have a feather-like shape and are single crystals. This morphology ensures that all the branches have a typical thickness of $\sim 40 \mathrm{~nm}$, but the whole particle size is about several micrometers, therefore being quite stable under electron beam irradiation (Figure $1 \mathrm{a}$ and $1 \mathrm{~b}) .{ }^{28}$ Ultrafast dynamics of the Fe L-edge EELS with an energy loss of over $700 \mathrm{eV}$ was probed by femtosecond convergent electron pulses after an ultrashort pumping with optical pulses $(\lambda=519 \mathrm{~nm}$; typically $12 \mathrm{~mJ} / \mathrm{cm}^{2}$ ). The probing size was about $400 \mathrm{~nm}$. When singleelectron packets are used in the stroboscopic mode to avoid the space-charge effect, an energy resolution as low as $\sim 0.63 \mathrm{eV}$ has been reached, ${ }^{29}$ but here, so as to allow for larger electron counts and a better signal to noise ratio, the single-electron condition was relaxed and all the measurements were taken at an energy resolution of $\sim 2 \mathrm{eV}$.

The temporal evolution of the FEELS for the core-loss spectra of haematite is displayed in Figure 2. Known as "white lines", ${ }^{30}$ which usually show in the $\mathrm{L}_{2,3}$ edge of transition metals when the $2 p$ core electrons are excited into well-defined unoccupied d states instead of a broad continuum, the $\mathrm{Fe}_{3}$-edge is much sharper and stronger than the $\mathrm{O} \mathrm{K}$-edge as exhibited in Figure 2c (with background subtracted). Analyzing the $\mathrm{Fe}_{3^{-}}$ edge is obviously a better choice than the $\mathrm{O} \mathrm{K}$-edge for a higher signal to noise ratio. As seen in Figure $2 \mathrm{a}$ and $2 \mathrm{~b}$, dramatic broadening and lowering of the $\mathrm{Fe}_{3}$-edge can be observed within the first picosecond after the $519 \mathrm{~nm}$ femtosecond laser pulse excitation (at time $\approx 0 \mathrm{ps}$ ), accompanied by a near full recovery in the next few picoseconds.

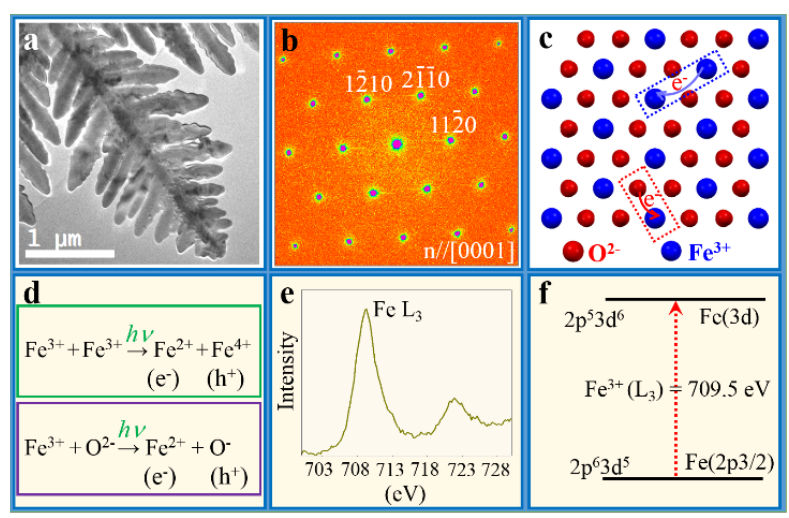

Figure 1. (a) TEM image and (b) selected area electron diffraction (SAED) pattern of the feather-like haematite single crystal. (c) Schematics for the two possible $\mathrm{Fe}^{3+}-\mathrm{Fe}^{3+}$ and $\mathrm{Fe}^{3+}-\mathrm{O}^{2-}$ charge transfer centers. (d) Possible charge transfer processes in haematite under $519 \mathrm{~nm}$ photoexcitation. (e,f) Fe L-edge EELS corresponding to the excitation of a Fe $2 p$ core electron of haematite to an unoccupied $3 \mathrm{~d}$ state with an energy change of over $700 \mathrm{eV}$.

It is noteworthy that the interaction between photons and the probing electrons near the surface of the sample, known as the foundation of photon-induced near field electron microscopy (PINEM) ${ }^{31-33}$ could be rather strong and hence leads to a significant broadening in the energy domain of the zero-loss probing electrons between -1 and +1 picosecond (Figure $\mathrm{S} 1$ ). When there are no intrinsic dynamics and the PINEM-electrons (the probing electrons which exchanged energy with photons) are uniformly distributed in space, convolution of the PINEMelectron-broadened zero-loss spectrum (ZLP) with the intrinsic Fe L3-edge would match the measured overall core-loss edge. However, the probing area in this work contains a blank gap area, where the average ratio of PINEM-electrons to the probing electrons could be slightly higher than that over the haematite particle probed (see Supporting Information). Since the ZLP is broadened by the PINEM-electrons generated across the entire probing area, and only the PINEM-electrons over the sample area contribute to the broadening of the core-loss edge, convolution of the PINEM-electron affected ZLP with the intrinsic Fe L3-edge could lead to a slight overestimation of the PINEMelectron induced core-loss edge broadening. If an approximation is made with ignoring such deviation, a comparison of the convolution of intrinsic Fe L3-edge before photoexcitation with the PINEM-electron unaffected ZLP and with the ZLP at 0.15 ps with strong PINEM-electron effect would demonstrate the broadening of the core-loss edge purely induced by PINEMelectrons. Figure 3 a shows that the PINEM-electron unaffected spectrum (green curve) is relatively sharp and high in intensity, and the simulation of the pure PINEM-electron effect results in a broadening (blue curve). However, the experimentally observed $\mathrm{Fe}_{3}$-edge at 0.15 ps shows further broadening (red curve), which implies that the PINEM-electrons cannot take the full responsibility for the total broadening. Another intrinsic broadening must be considered. 


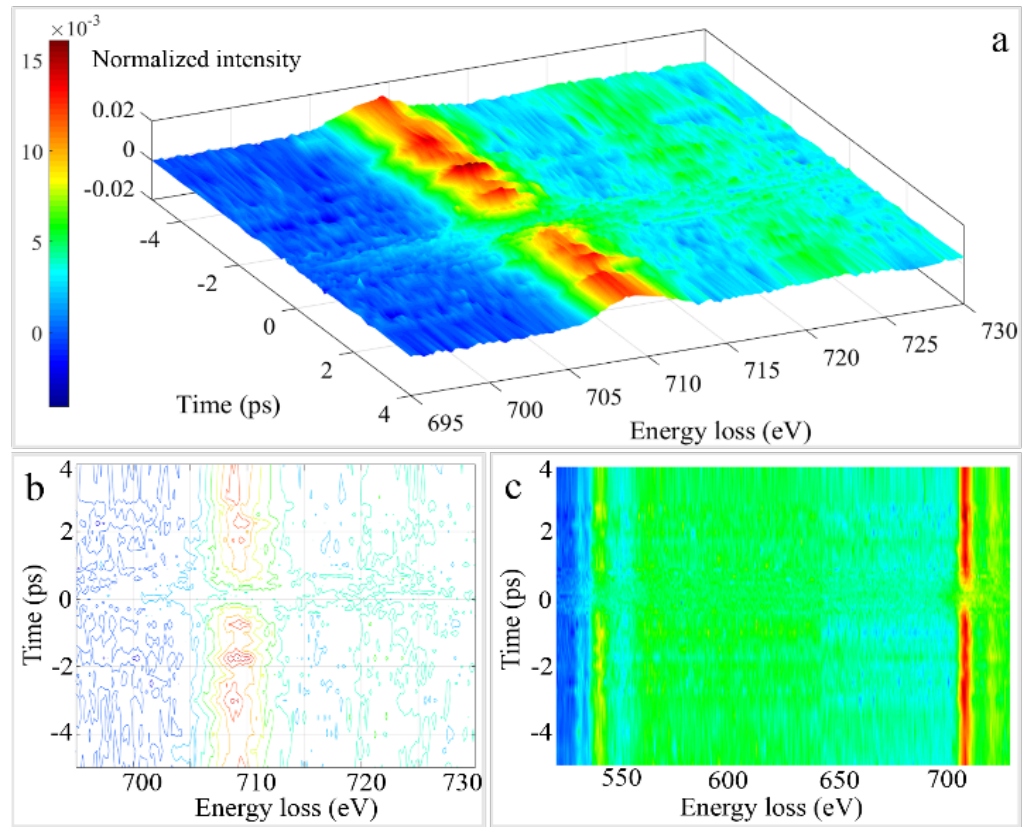

Figure 2. (a) 3D intensity-energy-time dynamics of the overall Fe L-edge FEELS. (b) Intensity contour map of (a). (c) Top view of Fe L3edge (above $700 \mathrm{eV}$ ) and $\mathrm{O} \mathrm{K}$-edge (around $540 \mathrm{eV}$ ) FEELS.

The intrinsic $\mathrm{Fe} \mathrm{L}_{3}$-edge before photoexcitation was then used to convolute with the time varying ZLP, and a pseudoVoigt function was used to determine the peak position and full width at half maximum (FWHM). The FWHM of the simulated $\mathrm{Fe}_{3}$-edges as a function of time was then compared with that of the experimentally observed overall $\mathrm{Fe}_{3}$-edges. As shown in Figure 3b, the maximum PINEM-electron-induced broadening (FWHM from 4.5 to $6.5 \mathrm{eV}$ ) is roughly equal with the intrinsic broadening (FWHM from 6.5 to $8.3 \mathrm{eV}$ ). The FWHM of $\mathrm{Fe} \mathrm{L}_{3}$-edge exhibited femtosecond dynamics, while no reliable temporal change for the peak position was detected.

The probing electron pulse has a width of about $500 \mathrm{fs}$, and the recorded $\mathrm{Fe}_{3}$-edges at a given time delay can be considered as the sum of many elementary $\mathrm{Fe}_{3}$-edges created by electrons passing through the sample in a series of time slices divided from the entire electron pulse. When excited by a shorter light pulse, these elementary $\mathrm{Fe}_{3}$-edges can have different inherent spectra, as the deviation of the measurement from the static PINEM-only simulation of the $\mathrm{Fe}_{3}$-edge displays femtosecond dynamics. Moreover, since the PINEM effect occurs only during the light pulse, the corresponding temporal slice of the evolving $\mathrm{L}_{3}$-edge will have relatively intense PINEM-electrons, while other parts of the spectrum from other time slices will have none. As a result, it is clear that it is not generally possible to model the effect of PINEM on a dynamically evolving spectrum by convolution with the ZLP.

In the present case, where the $\mathrm{L}_{3}$-edge displays excess broadening without displacement, if we take the above approximation of neglecting the error introduced by different PINEM-electron distributions in the blank gap area and the sample area, a modelled intrinsic edge at each delay can be obtained by fitting its convolution with the corresponding ZLP to the overall core-loss edge. This model edge should be no narrower than the elementary edge with least degree of broadening and no broader than the elementary edge with largest degree of broadening within the entire electron pulse, and might be considered qualitatively as a convolution based average. It should be borne in mind, however, that this procedure is limited in its application, and approximate in nature even for this special case, neglecting as it does the inhomogeneous distribution of PINEM electrons within the electron time pulse.

With this convolution based average method, intrinsic $\mathrm{Fe} \mathrm{L}_{3^{-}}$ edges within the PINEM-electron affected -1 to +1 picosecond regime were approximately extracted. Convolution of these with the PINEM-electron unaffected ZLP provides an approximation of the inherent dynamics of haematite. In this way, the dynamics of the FWHM of the $\mathrm{Fe}_{3}$-edge with PINEMelectron effect removed is shown in Figure $3 \mathrm{c}$. The error bar $( \pm$ $0.2 \mathrm{eV}$ ) is the measurement uncertainty as the spectra were recorded with an energy dispersion of $0.2 \mathrm{eV} /$ channel. Additional errors could be introduced while extracting the PINEM-electron effect which might be even greater than the measurement error. It should be noted that the time delays have a maximum possible uncertainty of $\pm 250 \mathrm{fs}$ considering the multiple acquisitions taken. ${ }^{34}$ Generally, after the initial fast rise, the FWHM of Fe $\mathrm{L}_{3}$-edge recovers within a few picoseconds.

The question now arises: what causes the femtosecond broadening of the $\mathrm{Fe}_{3}$-edge? The laser induced heating usually starts to cool down at a time delay of nanosecond scale, which finishes in a microsecond range. Therefore, the heating effect is neglected. ${ }^{5}$ An obvious answer is that the femtosecond dynamics should reflect the ultrafast generation and recombination of photoexcited carriers. 


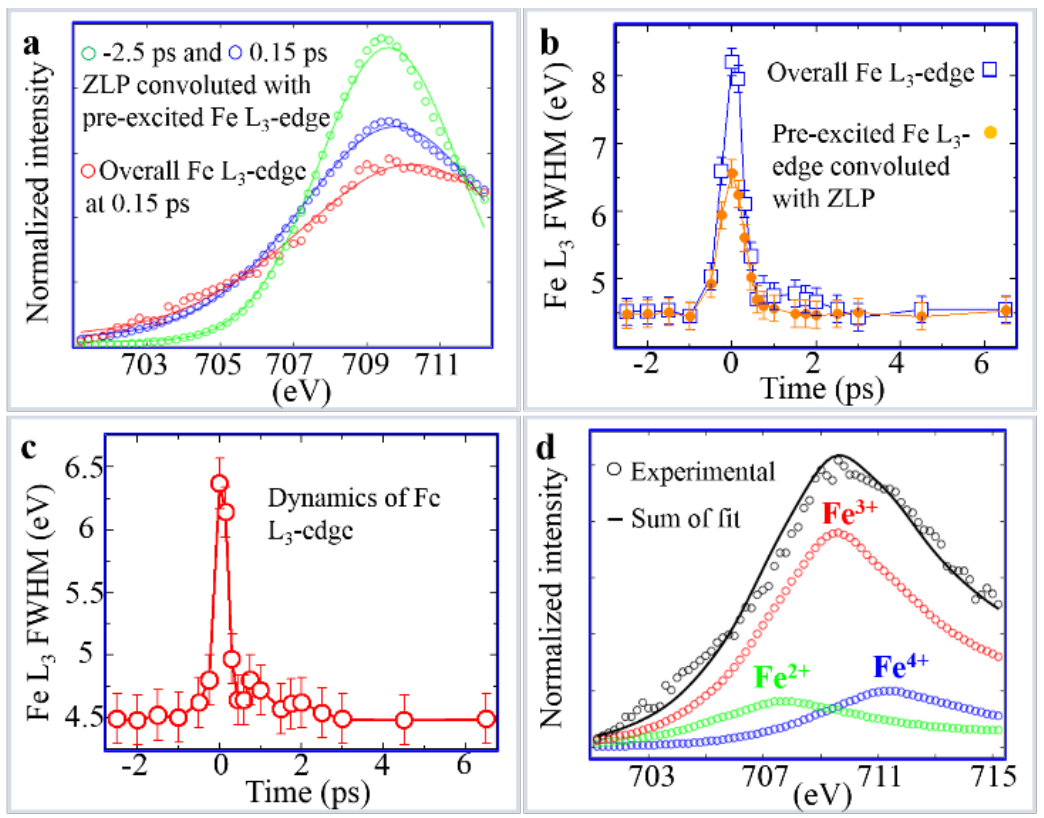

Figure 3. (a) A comparison of the $\mathrm{Fe}_{3}$-edge convoluted with the zero-loss spectra (ZLP) at negative time (no PINEM-electron effect, green circles), and at $0.15 \mathrm{ps}$ (strong PINEM-electron effect, blue circles), with the experimentally observed overall Fe $\mathrm{L}_{3}$-edge at $0.15 \mathrm{ps}$ (red circles). The solid lines are simulation fits using a pseudo-Voigt function to determine the peak position and FWHM. (b) Time dependent FWHM of the observed overall $\mathrm{Fe}_{3}$-edge and the $\mathrm{Fe}_{3}$-edge before laser excitation, convoluted with the ZLP changing with time. In the latter, the broadening of the edge is contributed only by the PINEM-electron effect within -1 to +1 ps regime. (c) Qualitative estimate of the intrinsic femtosecond dynamics of the FWHM of Fe $\mathrm{L}_{3}$-edge. (d) The comparison of the experimentally obtained overall Fe $\mathrm{L}_{3}$-edge at 0.15 ps with fitted spectra on the assumption of a static specimen with $32 \%$ of $\mathrm{Fe}^{3+}$ ions changed to equal amount of $\mathrm{Fe}^{2+}$ and $\mathrm{Fe}^{4+}$ ions, where the $\mathrm{Fe}_{3}$-edges for $\mathrm{Fe}$ cations with different oxidation states were convolved with the overall PINEM-electron-broadened zero loss spectrum at $0.15 \mathrm{ps}$.

Previous XAS study ${ }^{35}$ on the K-edge of vanadium oxide series from $\mathrm{VO}$ to $\mathrm{V}_{2} \mathrm{O}_{5}$ showed that the energy position shifted to higher energy with increasing formal oxidation state of vanadium atom in a linear manner, following the so-called Kunzl's law. ${ }^{36}$ Recent EELS study ${ }^{37}$ on the L-edge of a wide range of V, Mn- and Fe-based oxides reported similar dependence of chemical shift on oxidation state. The energy of $\mathrm{Fe}_{2}$-edge is known to shift from $\sim 707.8 \mathrm{eV}$ for $\mathrm{Fe}^{2+}$ in $\mathrm{FeO}$ to $\sim 709.5 \mathrm{eV}$ for $\mathrm{Fe}^{3+}$ in $\mathrm{Fe}_{2} \mathrm{O}_{3}{ }^{38}$ Although no data for $\mathrm{Fe}^{4+}$ in binary iron oxides are available, it is reasonable to expect, according to Kunzl's law, a blue shift of $\sim 1.7 \mathrm{eV}$ from $\mathrm{Fe}^{3+}$.

We next consider the two possible photoexcited charge transfer processes illustrated in Figure 1. If the direct ligand to metal $(\mathrm{O} 2 \mathrm{p} \rightarrow \mathrm{Fe} 3 \mathrm{~d}$ ) charge transfer transition dominates the $519 \mathrm{~nm}$ photoexcitation of haematite, the $\mathrm{Fe}_{3}$-edge will have a maximum broadening of $\sim 1.0 \mathrm{eV}$ in FWHM, and a red shift of $\sim 0.7$ $\mathrm{eV}$ when half of the $\mathrm{Fe}^{3+}$ cations are being reduced to $\mathrm{Fe}^{2+}$ cations. However, in the present work, a maximum broadening of over $1.8 \mathrm{eV}$ was measured and no detectable red shift was recorded throughout the experimental time scale. Therefore, it is more likely that the $\mathrm{Fe} 3 \mathrm{~d}$ to Fe $3 \mathrm{~d}$ charge transfer process dominates, and that the red shift of the $\mathrm{Fe}_{3}$-edge induced by the photogenerated $\mathrm{Fe}^{2+}$ is offset by the same amount of $\mathrm{Fe}^{4+}$ created, thus only leading to a broadening of the $\mathrm{Fe} \mathrm{L}_{3}$-edge. With a penetration depth of $\sim 50 \mathrm{~nm}$ in haematite ${ }^{21,22}$ for the $519 \mathrm{~nm}$ excitation, assuming a linear dependence of the photon absorption on the sample thickness, about $80 \%$ of the incident photons were absorbed by the $40 \mathrm{~nm}$-thick sample. The atomic density of iron in haematite, (crystal structure: Trigonal space group Rc, $a=5.038 \AA, c=13.772 \AA)$, is $\sim 0.0396$ per $\AA^{3}$. If each of the absorbed photons creates one $\mathrm{Fe}^{2+}-\mathrm{Fe}^{4+}$ electron-hole pair, the $519 \mathrm{~nm}$ optical pulse with a fluence of $12 \mathrm{~mJ} / \mathrm{cm}^{2}$, corresponding to 3.135 photons per $\AA^{2}$, will lead to the generation of $\mathrm{Fe}^{2+}$ and $\mathrm{Fe}^{4+}$ cations with a ratio of $\mathrm{Fe}^{2+}: \mathrm{Fe}^{4+}: \mathrm{Fe}^{3+}$ (remaining) $=$ $0.16: 0.16: 0.68$, which is well supported by the reasonable match between the sum of fitting with the experimental data of the $\mathrm{Fe}_{3}$-edge at 0.15 ps (Figure $3 \mathrm{~d}$ ), (neglecting differences in the inelastic scattering cross-section due to valence state and the effect of temporal evolution of the populations during the electron pulse).

Since the spin and Laporte selection rules for the Fe d-d electronic transitions can be relaxed by the strong magnetic coupling of electron spins of next-nearest neighboring $\mathrm{Fe}^{3+}$ cations and the hybridization between the $\mathrm{Fe} 3 \mathrm{~d}$ and $\mathrm{O} 2 \mathrm{p}$ orbitals in haematite,$^{39,40}$ the Fe $3 d-3 d$ transitions can take place with a considerable probability. Two photoinduced electron transfer processes might account for the generation of $\mathrm{Fe}^{2+}-\mathrm{Fe}^{4+}$ electron-hole pairs after the femtosecond $519 \mathrm{~nm}$ excitation, as illustrated in Figure 4. First, both of the two magnetically-coupled $\mathrm{Fe}^{3+}$ cations can be simultaneously excited to the ${ }^{4} \mathrm{~T}_{1}\left({ }^{4} \mathrm{G}\right)$ state from the ${ }^{6} \mathrm{~A}_{1}$ ground state by the $519 \mathrm{~nm}$ pulse. Electron transfer from one $\mathrm{Fe}^{3+}$ cation to the other can then happen in a manner of superexchange or hopping via the bridge oxygen anions. Also, the $3 \mathrm{~d}-3 \mathrm{~d}$ transition can happen only in a single cation of the pair. In this case, the energy of the pump photons is high enough to draw electrons jumping from the valence band to the conduction band. The excited electrons can also transfer to the other $\mathrm{Fe}^{3+}$ cation via the superexchange or hopping mechanism, ${ }^{41,42}$ producing $\mathrm{Fe}^{2+}-\mathrm{Fe}^{4+}$ electron-hole pairs. In the literature, ${ }^{39,40}$ the absorption of $519 \mathrm{~nm}$ by iron oxides was attributed 
to the below-bandgap pair excitation of $\left(\mathrm{Fe}^{3+}-\mathrm{Fe}^{3+}\right):{ }^{6} \mathrm{~A}_{1}+{ }^{6} \mathrm{~A}_{1}$ $\rightarrow{ }^{4} \mathrm{~T}_{1}\left({ }^{4} \mathrm{G}\right)+{ }^{4} \mathrm{~T}_{1}\left({ }^{4} \mathrm{G}\right)$, and the tail of across-bandgap $3 \mathrm{~d}-3 \mathrm{~d}$ transitions ${ }^{6} \mathrm{~A}_{1} \rightarrow{ }^{4} \mathrm{E},{ }^{4} \mathrm{~A}_{1}\left({ }^{4} \mathrm{G}\right)$, as well as the ligand-to-metal charge transfer. The experimental result from the present work, that the photoholes are mainly $\mathrm{Fe}^{4+}$, implies that the pair excitation generating $\mathrm{Fe}^{2+}-\mathrm{Fe}^{4+}$ electron-hole pairs plays a major role under $519 \mathrm{~nm}$ femtosecond pulses. Therefore, although the ligand-tometal charge transfer might be comparable to or even more significant than the across-bandgap Fe $3 d-3 d$ transition, the dominant photoholes detected in the experiment are $\mathrm{Fe}^{4+}$ cations.

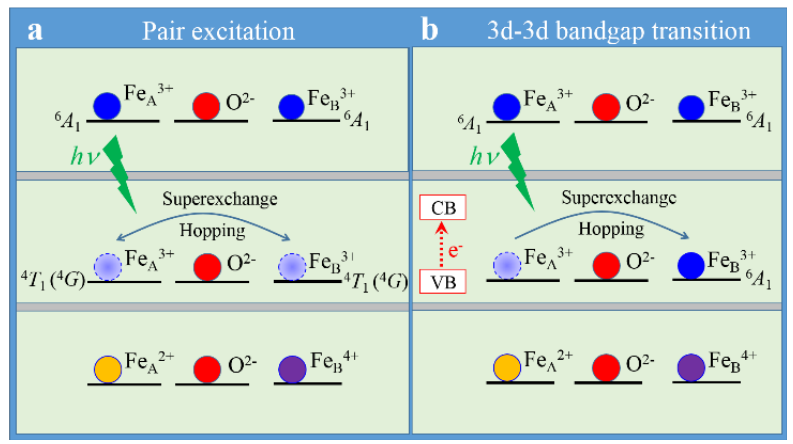

Figure 4. Schematics for the possible electron transfer processes between a pair of magnetically coupled neighboring $\mathrm{Fe}^{3+}$ cations under $519 \mathrm{~nm}$ excitation, in case of (a) pair excitation with two below-bandgap $\mathrm{Fe}$ 3d-3d transitions, and (b) single across-bandgap Fe 3d-3d transition.

The FEELS study here is sensitive to the oxidation state of iron cations and the photogenerated $\mathrm{Fe}^{2+}-\mathrm{Fe}^{4+}$ electron-hole pairs are revealed to be fully recombined in $\sim 3 \mathrm{ps}$. In contrast, in the previous TAS studies ${ }^{13}$ of haematite pumped by $400 \mathrm{~nm}$ optical pulses, where the $\mathrm{O} 2 \mathrm{p}$ to Fe $3 \mathrm{~d}$ ligand-to-metal charge transfer process dominated, ${ }^{17}$ the carrier recombination process can take up to hundreds of picoseconds. We show here that, under $519 \mathrm{~nm}$ excitation, the Fe d-d transitions dominate and the electron back transfer process, from $\mathrm{Fe}^{2+}$ cations to $\mathrm{Fe}^{4+}$ cations, is accomplished in $\sim 3 \mathrm{ps}$, although the resulting $\mathrm{Fe}^{3+}$ cations might stay at mid-bandgap states or trap states for much longer time before returning to the ground state (which would not be reflected in our measurements). The much shorter lifetime and the related shorter diffusion length of $\mathrm{Fe}^{4+}$ holes compared with the $\mathrm{O}^{-}$holes generated by $400 \mathrm{~nm}$ excitation can well explain the much higher photocatalytic performance during the photooxidation of water by haematite under $400 \mathrm{~nm}$ than $519 \mathrm{~nm}$ illumination in previous experiments, ${ }^{21,22}$ despite the similar absorption coefficients of haematite for the two wavelengths.

Femtosecond nanoscale elemental and oxidation-state mapping. The femtosecond broadening and lowering of the amplitude of the $\mathrm{Fe}_{3}$-edge, induced by the change of oxidation state and by the PINEM-electron effect with roughly equal contributions, make it possible to picture such transition on the femtosecond scale using the atom-selected elemental mapping by 4 D-EFTEM, as illustrated in Figure S2.

Time-dependent iron mapping was performed by using a three-window method with two pre-edge images and one postedge image, where the background contribution to the Fe postedge images was subtracted by extrapolating a power law function from the two pre-edge images (Figure 5a). To enable the mapping of the oxidation state change, a very narrow energy slit of $4 \mathrm{eV}$ was used for each window, over 5 fold narrower than the typical value of $20-50 \mathrm{eV}$ used when no such high energy resolution is needed. Such a narrow energy slit entails less electron counts, longer acquisition time and subsequent specimen drifting. A CCD binning of $8 \times 8$ instead of $1 \times 1$ was used to deposit more electron counts per pixel with the same acquisition time, which allowed us to take an energy-filtered image with a sufficiently high signal to noise ratio in $\sim 5 \mathrm{~min}$, thereby limiting the possible specimen drift to $<2.5 \mathrm{~nm}$ in our 4D-EFTEM system. This degree of specimen drifting does not have a pronounced effect on the image quality at the working magnification, where a single pixel covers over $10 \mathrm{~nm}$ in space. The energy window of the Fe post-edge was centred at $709.5 \mathrm{eV}$, the peak energy loss of $\mathrm{Fe}^{3+} \mathrm{L}_{3}$-edge, which can maximize the temporal change induced by the change of the Fe oxidation state from $3+$ to $2+$ and $4+$.

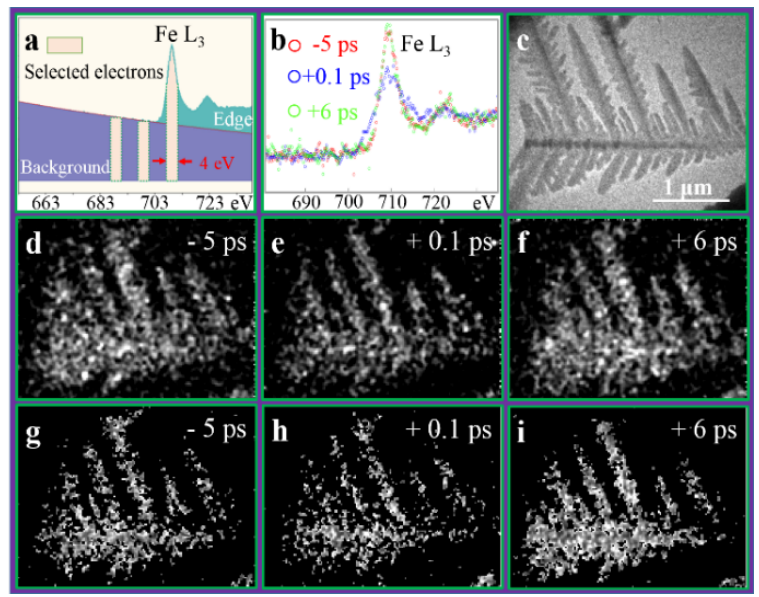

Figure 5. (a) Electrons centred at 709.5, 699.5 and $689.5 \mathrm{eV}$ energy loss are selected by a slit window of $4 \mathrm{eV}$ for the elemental mapping and the jump-ratio images. (b) Time-dependent EELS of $\mathrm{Fe} \mathrm{L}_{3}$-edge with background subtracted. (c) Ultrafast TEM image of a typical $\mathrm{Fe}_{2} \mathrm{O}_{3}$ particle without energy filtering. Temporal change of iron elemental mapping images (d-f) and jump-ratio images (g-i) of the $\alpha-\mathrm{Fe}_{2} \mathrm{O}_{3}$ particle obtained by a three-window method with two pre-edge $(699.5 \pm 2 \mathrm{eV}, 689.5$ $\pm 2 \mathrm{eV})$ and one post-edge $(709.5 \pm 2 \mathrm{eV})$, and a two-window method with one pre-edge $(699.5 \pm 2 \mathrm{eV})$ and one post-edge $(709.5 \pm 2 \mathrm{eV})$, respectively.

Figure 5 shows the time-resolved iron elemental mapping of a typical haematite particle as well as the ultrafast TEM image without energy filtering. The post-edge image was taken using inelastic electrons with energy loss of $709.5 \pm 2 \mathrm{eV}$, while the two pre-edge images used electrons with energy loss centred at 699.5 and $689.5 \mathrm{eV}$ respectively (Figure 5a). Consistent with the femtosecond lowering of the $\mathrm{Fe}^{3+} \mathrm{L}_{3}$ peak at $709.5 \mathrm{eV}$ revealed by the ultrafast EELS, the $\mathrm{Fe}^{3+} \mathrm{L}_{3}$ image shows $\mathrm{a} \sim 20 \%$ decrease in electron counts at $\sim 100$ fs after the femtosecond photoexcitation, which recovers within 6 ps (Figure 5b). Besides, a two-window method dividing the post-edge image $(709.5 \pm 2 \mathrm{eV})$ by a pre-edge image $(699.5 \pm 2 \mathrm{eV})$ was used to create the so-called "jump-ratio" image, which shows a better signal to noise ratio and better contrast than direct elemental mapping. Similarly, the ultrafast decrease and recovery of the sum of the jump-ratio value also mapped out the temporal change of the $\mathrm{Fe}$ oxidation state induced by the femtosecond 
$519 \mathrm{~nm}$ excitation. The feather-like nanostructures of the haematite particle are well resolved in all these images thanks to the high spatial resolution of the 4D elemental mapping technique that we have developed.

\section{CONCLUSION}

In summary, our ability to record high resolution (in energy, space and time) 4D-elemental mapping based on FEELS makes it possible to obtain both in-situ nanoscale elemental and oxidation-state mapping by atom-selected imaging. Moreover, this is done with unprecedented time resolution and eV energy resolution, covering the energy loss range of up to $730 \mathrm{eV}$. Compared with previous report on the 4D visualization of charge transfer in reciprocal space by ultrafast electron diffraction, ${ }^{34}$ the $4 \mathrm{D}$ elemental mapping technique developed here makes the imaging of electronic transition and charge transfer with high spatiotemporal resolution possible in real space.

Our studies of the photoexcitation of the binary transitionmetal oxide, haematite, by a femtosecond laser pulse at a wavelength of $519 \mathrm{~nm}$ has enabled us to resolve the question of the nature of the photoinduced electronic holes, thereby elucidating the questions raised by prior theoretical calculations. As a result of the unprecedented insights that we have been able to deploy, we can distinguish two types of holes, i.e. $\mathrm{Fe}^{4+}$ and $\mathrm{O}^{-}$. The $\mathrm{Fe}^{4+}$ cations are identified by EELS and are shown to exhibit a lifetime of a few picoseconds, much shorter than the life time of $\mathrm{O}^{-}$ holes generated by $400 \mathrm{~nm}$ excitation, and hence explain the much better performance in photooxidation of water by haematite photoanode under $400 \mathrm{~nm}$ illumination than under the green lighting in previous reports. This work should open the way for future investigations that entail ultrafast, atomic-scale visualization of charge transfer in real-space and atom-selective imaging of a variety of elements in vitally important inorganic photocatalysts. It is conceivable that such new information as we have uncovered here may also be accessed by a combination of ultrafast laser spectroscopy and/or scanning ultrafast electron microscopy.

\section{EXPERIMENTAL SECTION}

Sample preparation. Briefly, feather-like haematite particles were grown by the hydrolysis of $\left[\mathrm{Fe}(\mathrm{CN})_{6}\right]^{3-}$ anions. Firstly, $\mathrm{K}_{3} \mathrm{Fe}(\mathrm{CN})_{6}$ was dissolved in deionized water with a concentration of $10 \mathrm{mM}$. Then the solution was subsequently sealed in an autoclave, and maintained at $180{ }^{\circ} \mathrm{C}$ for $48 \mathrm{~h}$, followed by cooling down to room temperature gradually. ${ }^{28}$

The particles were then dispersed in ethanol, and a small drop of the suspension was deposited onto a graphene thin film coated 2,000 mesh copper grid. When dried in air, it was ready for the electron microscopic analysis. The sample was measured in the vacuum of EM, and was not in contact with a liquid electrolyte.

Apparatus and data acquisition. Caltech's second-generation ultrafast transmission electron microscope (UEM-2) was used, the principle of which has been described elsewhere. ${ }^{43}$ Briefly, a 259-nm fs pulse was used to extract the probing photoelectrons from the $\mathrm{LaB}_{6}$ cathode, and a $519-\mathrm{nm}$ fs pulse was used as pump beam and guided onto the specimen by a series of mirrors. The temporal delay between the electron-pulse and laser excitation can be adjusted as needed by varying the optical delay line between the two laser pulses. The equipped Gatan post-column energy filter, GIF Tridiem, enables the capture of EELS and EFTEM data.

A repetition rate of $500 \mathrm{KHz}$ was used for the FEELS measurement. Each core-loss spectrum was recorded with an energy dispersion of $0.2 \mathrm{eV} / \mathrm{channel}$, and was taken in a single acquisition with an integration time of $\sim 10 \mathrm{~min}$. The diameter of the spectrometer entrance aperture was set at $5 \mathrm{~mm}$. Since the system instability can cause gradual energy drift up to several $\mathrm{eV} / \mathrm{h}$, low-loss spectra including the zero-loss peak were recorded before and after each core-loss spectrum acquisition for energy correction. Switching between the low-loss and core-loss energy windows was achieved by changing the drift-tube voltage, which has a fast response without any hysteresis.

In the EFTEM measurement, the repetition rate was set as 1 $\mathrm{MHz}$ which produced more electrons with a given acquisition time. Accordingly, the fluence of the excitation dropped from $12 \mathrm{~mJ} / \mathrm{cm}^{2}$ at $500 \mathrm{KHz}$ to about $5 \mathrm{~mJ} / \mathrm{cm}^{2}$. The $4 \mathrm{eV}$ energy slit was chosen as a compromise between the energy resolution and electron counts. Narrower energy slit has better energy resolution but the signal to noise ratio is too low, while a broader energy slit loses the energy resolution necessary to map out iron oxidation-state change in haematite. The elemental mapping and the jump-ratio images were processed using the Gatan Digital Micrograph software package.

\section{ASSOCIATED CONTENT}

\section{SUPPORTING INFORMATION}

The Supporting Information is available free of charge on the ACS Publications website at DOI:

Figure S1, PINEM-electron effect on the zero-loss EELS spectra and ultrafast TEM images of haematite. Figure S2, Ultrafast iron oxidation-state and elemental mapping.

\section{AUTHOR INFORMATION}

Corresponding authors

$$
\begin{aligned}
& \text { *jmt2@cam.ac.uk } \\
& \text { *wzhou@st-andrews.ac.uk }
\end{aligned}
$$

ORCID

Wuzong Zhou: 0000-0001-9752-7076

Notes

The authors declare no competing financial interest.

\section{ACKNOWLEDGEMENTS}

This work was supported by the Air Force Office of Scientific Research (FA9550-11-1-0055) in the Gordon and Betty Moore Center for Physical Biology at the California Institute of Technology. The studies described in this paper were conceived by Ahmed Zewail, and most of the insights that we reveal were greatly influenced by his input. We thank Prof. Nathan S. Lewis at the California Institute of Technology for helpful comments.

\section{REFERENCES}

(1) Egerton, R. F. Electron Energy-Loss Spectroscopy in the Electron Microscope; Springer, Science \& Business Media, New York, 2011; pp 293-301.

(2) Muller, D. A.; Kourkoutis, L. F.; Murfitt, M.; Song, J. H.; Hwang, H. Y.; Silcox, J.; Dellby, N.; Krivanek, O. L. Science 2008, 319, 1073-1076. 
(3) Zewail, A. H. Phil. Trans. R. Soc. A 2010, 368, 1191-1204.

(4) Thomas, J. M. Angew. Chem. Int. Ed. 2009, 48, 8824-8826.

(5) van der Veen, R. M.; Penfold, T. J.; Zewail, A. H. Struct. Dynamics 2015, 2, 024302.

(6) Khaselev, O.; Turner, J. A. Science 1998, 280, 425-427.

(7) Hu, S.; Shaner, M. R.; Beardslee, J. A.; Lichterman, M.; Brunschwig, B. S.; Lewis, N. S. Science 2014, 344, 1005-1009.

(8) Shaner, M. R.; Atwater, H. A.; Lewis, N. S.; McFarland, E. W. Energy Environ. Sci. 2016, 9, 2354-2371.

(9) Sivula, K.; Le Formal, F.; Grätzel, M. ChemSusChem 2011, 4, 432-449.

(10) Golnak, R.; Bokarev, S. I.; Seidel, R.; Xiao, J.; Grell, G.; Atak, K.; Unger, I.; Thürmer, S.; Aziz, S. G.; Kühn, O.; Winter, B.; Aziz, E. F. Sci. Reports 2016, 6, 24659.

(11) Kay, A.; Cesar, I.; Grätzel, M. J. Am. Chem. Soc. 2006, 128, 15714-15721.

(12) Cherepy, N. J.; Liston, D. B.; Lovejoy, J. A.; Deng, H.; Zhang, J. Z. J. Phys. Chem. B 1998, 102, 770-776.

(13) Sorenson, S.; Driscoll, E.; Haghighat, S. J.; Dawlaty, M. J. Phys. Chem. C 2014, 118, 23621-23626.

(14) Milne, C. J.; Penfold, T. J.; Chergui, M. Coord. Chem. Rev. 2014, 277-278, 44-68.

(15) Silatani, M.; Lima, F. A.; Penfold, T. J.; Rittman, J.; Reinhard, M. E.; Rittmann-Frank, H. M.; Borca, C.; Grolimund, D.; Milne, C. J.; Chergui, M. PNAS 2015, 112, 12922-12927.

(16) Huse, N.; Cho, H.; Hong, K.; Jamula, L.; de Groot, F. M. F.; Kim, T. K.; McCusker, J. K.; Schoenlein, R. W. J. Phys. Chem. Lett. 2011, 2, 880-884.

(17) Vura-Weis, J.; Jiang, C. M.; Liu, C.; Gao, H.; Lucas, J. M.; de Groot, F. M. F.; Yang, P.; Alivisatos, A. P.; Leone, S. R. J. Phys. Chem. Lett. 2013, 4, 3667-3671.

(18) Katz, J. E.; Gilbert, B.; Zhang, X. Y.; Attenkofer, K.; Falcone, R. W.; Waychunas, G. A. J. Phys. Chem. Lett. 2010, 1, 1372-1376.

(19) Katz, J. E.; Zhang, X. Y.; Attenkofer, K.; Chapman, K. W.; Frandsen, C.; Zarzycki, P.; Rosso, K. M.; Falcone, R. W.; Waychunas, G. A.; Gilbert, B. Science 2012, 337, 1200-1203.

(20) Santomauro, F. G.; Lübcke, A.; Rittmann, J.; Baldini, E.; Ferrer, A.; Silatani, M.; Zimmermann, P.; Grübel, S.; Johnson, J. A.; Mariager, S. O.; Beaud, P.; Grolimund, D.; Borca, C.; Ingold, G.; Johnson, S. L.; Chergui, M. Sci. Reports 2015, 5, 14834.

(21) Kennedy, J. H.; Frese, K. W. J. Electrochem. Soc. 1978, 125, $709-714$.

(22) Kim, D. W.; Riha, S. C.; Demarco, E. J.; Martinson, A. B. F.; Farha, O. K.; Hupp, J. T. ACS Nano 2014, 8, 12199-12207.

(23) Catti, M.; Valerio, G.; Dovesi, R. Phys. Rev. B: Condens. Matter Mater. Phys. 1995, 51, 7441-7450.

(24) Butler, W. H.; Bandyopadhyay, A.; Srinivasan, R. J. Appl. Phys. 2003, 93, 7882-7884.

(25) Pailhe, N.; Wattiaux, A.; Gaudon, M.; Demourgues, A. J. Solid State Chem. 2008, 181, 2697-2704.

(26) Gaudon, M.; Pailhe, N.; Majimel, J.; Wattiaux, A.; Abel, J.; Demourgues, A. J. Solid State Chem. 2010, 183, 2101-2109.

(27) Barroso, M.; Pendlebury, S. R.; Cowan, A. J.; Durrant, J. R. Chem. Sci. 2013, 4, 2724-2734.

(28) Green, A. E.; Chiang, C.-Y.; Greer, H. F.; Waller, A.; Ruszin, A.; Webster, J.; Niu, Z. Y.; Self, K.; Zhou, W. Z. Crystal Growth \& Design 2017, 17, 800-808.

(29) Liu, H.; Baskin, J. S.; Zewail, A. H. PNAS 2016, 113, 2041-2046.

(30) Leapman, R. D.; Grunes, L. A.; Fejes, P. L. Phys. Rev. B 1982, $26,614-635$.

(31) Barwick, B.; Flannigan, D. J.; Zewail, A. H. Nature 2009, 462, 902-906.

(32) Zewail, A. H.; Thomas, J. M. 4D electron microscopy: imaging in space and time; Imperial College Press: London, 2010; pp 263.

(33) Yurtsever, A.; van der Veen, R. M.; Zewail, A. H. Science 2012, $335,59-64$

(34) Yoo, B-K.; Su, Z. X.; Thomas, J. M.; Zewail, A. H. PNAS 2016, $113,503-508$.
(35) Wong, J.; Lytle, F. W.; Messmer, R. P.; Maylotte, D. H. Phys. Rev. B 1984, 30, 5596-5610.

(36) Mande, C.; Sapre, V. B. in Advances in X-ray spectroscopy (Bonnelle, C.; Mande C. Eds.); Pergamon: New York, 1982, pp 287-301.

(37) van Aken, P. A.; Liebscher, B. Phys. Chem. Minerals 2002, 29, $188-200$.

(38) Tan, H.; Verbeeck, J.; Abakumov, A.; Tendeloo, G. V. Ultramicroscopy 2012, 116, 24-33.

(39) Sherman, D. M.; Waite, T. D. Am. Mineral. 1985, 70, 1262-1269.

(40) Borghi, E.; Occhiuzzi, M.; Foresti, E.; Lesci, I. G.; Roveri, N. Phys. Chem. Chem. Phys. 2010, 12, 227-238.

(41) Natali, M.; Campagna, S.; Scandola, F. Chem. Soc. Rev. 2014, 43, 4005-4018.

(42) Pavarini, E.; Koch, E.; Anders, F.; Jarrel, M. Modeling and Simulation; Forschungszentrum: Jüilich, 2012; Vol. 2, pp 7.13-7.17.

(43) Barwick, B.; Park, H. S.; Kwon, O. H.; Baskin, J. S.; Zewail, A. H. Science 2008, 322, 1227-1231. 
Ultrafast Elemental and Oxidation-state Mapping of Haematite by 4D Electron Microscopy

Zixue Bu, J. Spencer Baskin, Wuzong Zhou, John M. Thomas, and Ahmed H. Zewail

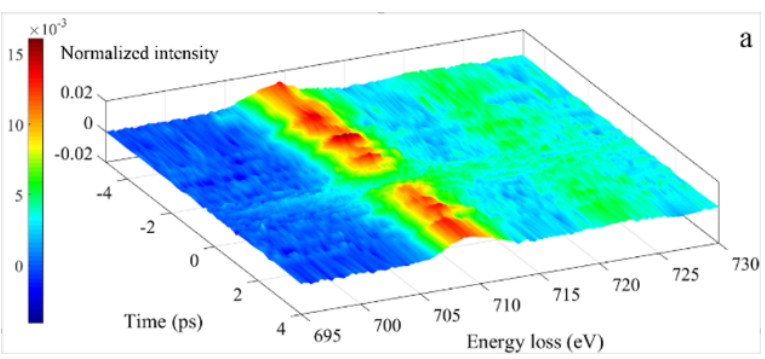

